\title{
Venous Obstruction of the Thigh
}
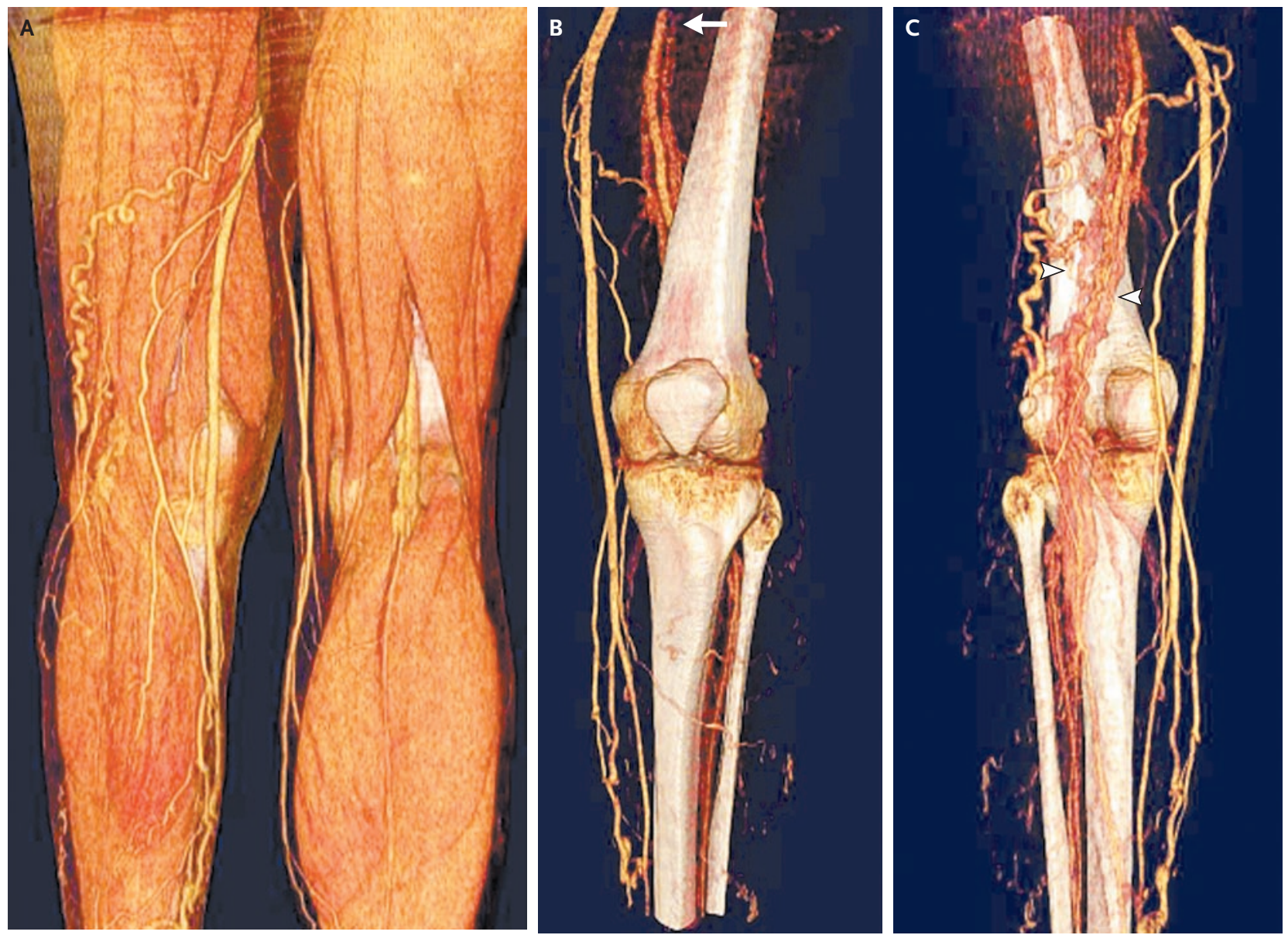

Alberto Caggiati, M.D. Carlo Catalano, M.D.

Sapienza University of Rome 00161 Rome, Italy

alberto.caggiati@uniromal.it
56-YEAR-OLD MAN PRESENTED WITH A POST-THROMBOTIC SYNDROME OF the left leg (involving edema, varicose veins, trophic skin changes, and an ulcer, $5 \mathrm{~cm}$ in diameter, of the distal medial leg), resulting from deep-vein thrombosis 2 years earlier. He had been found to have the factor V Leiden mutation and began receiving maintenance therapy with anticoagulants. Preoperative computed tomography of the leg was used to examine the superficial and deep venous systems, after the images were reformatted to eliminate the subcutaneous fat (Panel A) and muscles (Panels B and C, and see the video). The upper portion of the femoral vein was found to be occluded (Panel B, arrow). Distally, the femoral vein appeared to be duplicated, with the two branches forming an irregular plexus around the femoral artery (Panel C, arrowheads). Because of the persistence of the occlusion, no valvular reconstruction could be performed in the deep veins, and the main varicose trunks could not be surgically repaired. To improve healing and drainage from the ulcerated, dystrophic area of the skin, the distal varicosities were resected, and medicated compressive bandages were applied. The ulcer healed after 4 weeks, and the symptoms improved considerably. The patient has done well subsequently, receiving maintenance anticoagulant therapy and wearing compression stockings.

Copyright (c) 2009 Massachusetts Medical Society. 